\title{
Dimensional Reduction in Bose-Condensed Alkali-Metal Vapors
}

\author{
L. Salasnich ${ }^{1}$, A. Parola ${ }^{2}$ and L. Reatto ${ }^{1}$ \\ ${ }^{1}$ Istituto Nazionale per la Fisica della Materia, Unità di Milano, Dipartimento di Fisica, Università di Milano, \\ Via Celoria 16, 20133 Milano, Italy \\ ${ }^{2}$ Istituto Nazionale per la Fisica della Materia, Unità di Como, Dipartimento di Scienze Fisiche, Università dell'Insubria, \\ Via Valeggio 11, 23100 Como, Italy
}

\begin{abstract}
We investigate the effects of dimensional reduction in atomic Bose-Einstein condensates (BECs) induced by a strong harmonic confinement in the cylindric radial direction or in the cylindric axial direction. The former case corresponds to a transition from 3D to 1D in cigar-shaped BECs, while the latter case corresponds to a transition from 3D to 2D in disc-shaped BECs. We analyze the first sound velocity in axially-homogeneous cigar-shaped BECs and in radially-homogeneous disc-shaped BECs. We consider also the dimensional reduction in a BEC confined by a harmonic potential both in the radial direction and in the axial direction. By using a variational approach, we calculate monopole and quadrupole collective oscillations of the BEC. We find that the frequencies of these collective oscillations are related to the dimensionality and to the repulsive or attractive inter-atomic interaction.
\end{abstract}

PACS Numbers: 03.75.Kk

In the experiments with Bose-Einstein condensates (BECs) the atomic cloud is confined by a magnetic or magneto-optical trap. In the simplest case the confining field acts as an anisotropic harmonic potential. Recent experiments $[1,2]$ have shown that it is possible to change the frequencies of the harmonic confinement inducing a dimensional reduction of the system: from 3D to $1 \mathrm{D}$ and from $3 \mathrm{D}$ to $2 \mathrm{D}$. Effects of reduced dimension in BECs have been theoretically investigated by many authors [3-6], but less attention has been paid to the transition from a dimensional regime to another.

In this brief report we study in detail the dimensional reduction in BECs by using nonpolynomial Schrödinger equations, which are derived from the 3D Gross-Pitaevskii equation (GPE) [7]. In this way we calculate chemical potential, elementary excitations and first sound velocity for cigar-shaped and disc-shaped BECs. Moreover, we apply a variational technique [8] to obtain monopole and quadrupole collective excitations of the BEC with both positive and negative scattering length $a_{s}$. In particular, we analyze the effects of anisotropy and inter-atomic strength on these collective frequencies.

From $3 D$ to $1 D$. - We have recently shown that the dynamics of a cigar-shaped BEC of $N$ atoms, confined by a harmonic potential of frequency $\omega_{r}$ in the cylindric radial direction $(x, y)$ and by a generic potential $V(z)$ in the cylindric axial direction $z$, is well described by an effective 1D nonpolynomial Schrödinger equation (1D NPSE) for the axial wave function $f(z, t)$ of the BEC [7]. Within the 1D NPSE approach, the BEC is approximated by a Gaussian in the cylindric radial direction. The Gaussian width $\sigma$ is related to $f$ by $\sigma=\left(1+g N|f|^{2}\right)^{1 / 4}$, where $g=2 a_{s} / a_{r}$ is the effective interaction strength with $a_{r}=\sqrt{\hbar /\left(m \omega_{r}\right)}$ the characteristic harmonic length in the radial direction [7]. Note that we write length is in units $a_{r}$ and energy in units $\hbar \omega_{r}$.
In the limit of weak radial confinement, which corresponds to the 3D regime, the axial density $N|f|^{2}$ satisfies the condition $g N|f|^{2}>>1$ and the 1D NPSE reduces to a 1D nonlinear Schrödinger equation with quadratic nonlinearity. In the opposite limit $g N|f|^{2}<<1$ of strong radial confinement, which corresponds to a $1 \mathrm{D}$ regime, the 1D NPSE becomes a 1D nonlinear Schrödinger equation with cubic nonlinearity, also called 1D GPE [4]. It is important to remember that the 1D NPSE is derived from the 3D GPE, but the 3D GPE (and also 1D NPSE) is no more valid in the limit of strong diluteness: in this case the atomic cloud becomes a Tonks gas, i.e. a gas of impenetrable Bosons [9]. In our units the system is far from the Tonks regime if the condition $g N|f|^{2} \gg g^{2}$ is satisfied [10].

In previous papers [7] we have verified that the $1 \mathrm{D}$ NPSE is able to capture the full 3D regime, the full $1 \mathrm{D}$ regime (apart the Tonks gas) and the region in between. Here we show that an analytical expression for the shape of the axial density can be obtained by neglecting the kinetic term, the so-called Thomas-Fermi (TF) approximation, in the 1D NPSE. Then, setting $f(z, t)=e^{-i \mu t} f_{0}(z)$ one finds for the axial density profile $\rho_{1}(z)=N\left|f_{0}(z)\right|^{2}$ the following expression

$$
\rho_{1}(z)=\frac{2}{9 g}\left[\mu(z)^{2}-3+\mu(z) \sqrt{\mu(z)^{2}+3}\right],
$$

where $\mu(z)=\mu-V(z)$ with $\mu$ the chemical potential. This density profile reduces to $\rho_{1}(z)=(2 \mu(z))^{2} /(9 g)$ when $g \rho_{1}>>1$, that is very close (with the factor $4 / 9$ instead of $1 / 2$ ) to the axial density profile of the TF 3D regime obtained from the 3D GPE. Eq. (1) reduces to $\rho_{1}(z)=(\mu(z)-1) / g$ when $g \rho_{1}<<1$, that is exactly the density profile of the TF $1 \mathrm{D}$ regime obtained from $1 \mathrm{D}$ GPE.

In the case $V(z)=0$, which corresponds to a homogeneous $\mathrm{BEC}$ in the $z$ direction with periodic boundary 
conditions, one gets the axial velocity of first sound as $c_{1}=\left(\rho_{1} \frac{\partial \mu}{\partial \rho_{1}}\right)^{1 / 2}$ form which one finds

$$
c_{1}=\sqrt{\frac{5}{4} \frac{g \rho_{1}}{\sqrt{1+g \rho_{1}}}-\frac{1}{4} \frac{g \rho_{1}+2 g^{2} \rho_{1}^{2}}{\left(1+g \rho_{1}\right)^{3 / 2}}} .
$$

In Figure 1 we plot $c_{1}$ as a function of $g \rho_{1}$. The figure shows that $c_{1}$ strongly depends on the dimensionality. In fact, our expression for the velocity of sound gives $c_{1}=\sqrt{3 / 4}\left(g \rho_{1}\right)^{1 / 4}$ in the $3 \mathrm{D}$ regime and it becomes $c_{1}=\sqrt{g \rho_{1}}$ in the $1 \mathrm{D}$ regime. Note that our $1 \mathrm{D}$ value for $c_{1}$ is in full agreement with the result of Menotti and Stringari [10], while at higher density our result $c_{1}$ differs from the exact one obtained by the 3D GPE [10], only for the constant that is $\sqrt{2} / 2$ instead of $3 / 4$. This small discrepancy (relative error less than 3\%) in the 3D regime is a consequence of using in the 1D NPSE a Gaussian radial wave function. Thus, our Eq. (2) for the sound velocity $c_{1}$ interpolates very well between the exact results of low density (1D regime) and high density (3D regime).

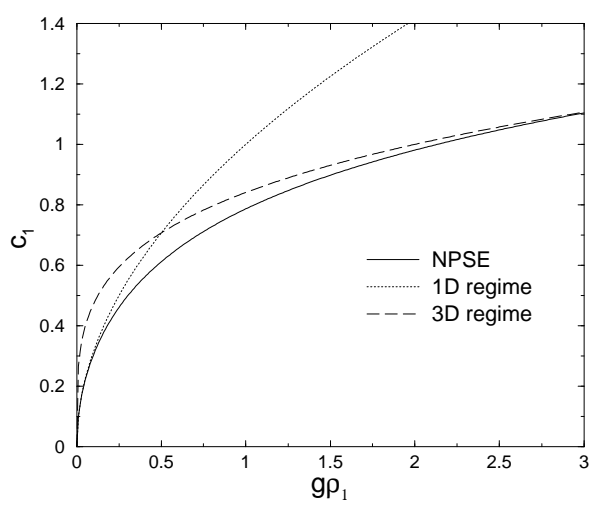

FIG. 1: First sound axial velocity $c_{1}$ versus inter-atomic strength $g \rho_{1}$, where $g=2 a_{s} / a_{r}$ with $a_{s}$ scattering length and $a_{r}$ radial harmonic length. $\rho_{1}$ is the axial density of the cigarshaped axially-homogeneous BEC. Length in units $a_{r}$, density in units $1 / a_{r}$ and time in units $1 / \omega_{r}$.

In the case $V(z)=m \omega_{z}^{2} z^{2} / 2$ the low-frequency elementary excitations, which are collective oscillation of the BEC, can be more easily calculated with a variational approach [8] from the full 3D GPE instead of using the 1D NPSE. The most natural choice is a Gaussian wave function with two variational parameters: the radial width $\sigma$ and the axial width $\eta$. Then the effective potential energy $U$ of the atomic cloud obtained from the 3D Gross-Pitaevskii energy functional is given by

$$
U=\frac{1}{\sigma^{2}}+\frac{1}{2 \eta^{2}}+\sigma^{2}+\frac{1}{2 \lambda^{2}} \eta^{2}+\frac{N g}{\sqrt{2 \pi}} \frac{1}{\sigma^{2} \eta},
$$

where $\lambda=\omega_{r} / \omega_{z}$ is the anisotropy parameter of the confining harmonic potential. With $\lambda>1$ the $\mathrm{BEC}$ is cigar-shaped, while for $\lambda<1$ the BEC is disc-shaped. The equilibrium point $\left(\sigma^{*}, \eta^{*}\right)$, corresponds to the minimum of the effective potential energy of the system.
The low-frequency collective excitations of the BEC are the small oscillations around the equilibrium point. For $\lambda=1$ these two normal mode frequencies can be identified as the monopole frequency $\omega_{M}$ and the quadrupole frequency $\omega_{Q}$ [8]. One finds that for $\lambda>>1$ the frequency $\omega_{M}$ is mainly transverse and the frequency $\omega_{Q}$ is mainly axial, while for $\lambda<<1$ the frequency $\omega_{M}$ is mainly axial and the frequency $\omega_{Q}$ is mainly transverse.

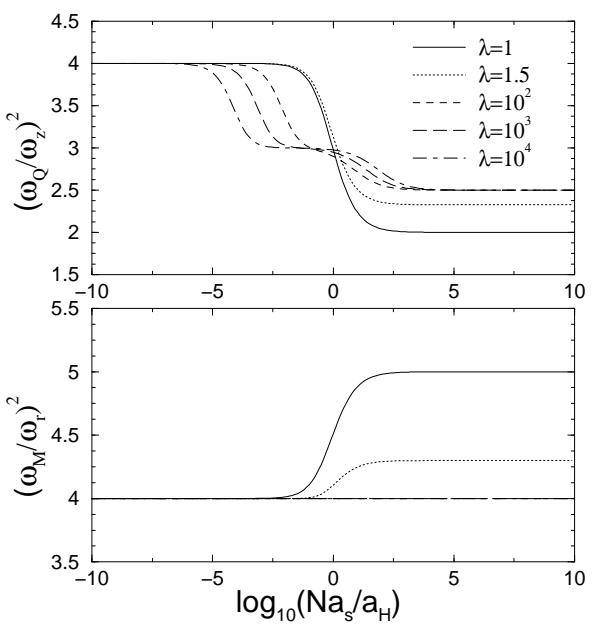

FIG. 2: Monopole frequency $\omega_{M}$ and quadrupole frequency $\omega_{Q}$ of collective oscillations for a cigar-shaped BEC. Case of positive scattering length $a_{s} . \lambda=\omega_{r} / \omega_{z}$ is the anisotropy of the harmonic trap. $a_{H}=\sqrt{\hbar /\left(m \omega_{H}\right)}$ with $\omega_{H}=\left(\omega_{r}^{2} \omega_{z}\right)^{1 / 3}$.

In Figure 2 we plot $\omega_{M}$ and $\omega_{Q}$ as a function of the inter-atomic strength for different values of the anisotropy parameter $\lambda$. In the spherical case $(\lambda=1)$ the monopole frequency $\omega_{M}$ ranges between the value $\left(\omega_{M} / \omega_{r}\right)^{2}=5$ of the 3D regime of mean-field bosons (isotropic TF 3D) and the value $\left(\omega_{M} / \omega_{r}\right)^{2}=4$ of quasiideal bosons. By increasing $\lambda$ the frequency $\omega_{M}$ becomes a constant, given by $\left(\omega_{M} / \omega_{r}\right)^{2}=4$, independently on the interaction strength.

The transition from 3D to $1 \mathrm{D}$ is even better shown by the quadrupole frequency $\omega_{Q}$ (see also [12]). In this case, for very large values of $\lambda$ the frequency $\omega_{Q}$ shows three plateaus: at $\left(\omega_{Q} / \omega_{z}\right)^{2}=5 / 2$ the BEC is in the $3 \mathrm{D}$ regime (anisotropic TF $3 \mathrm{D}$ ), at $\left(\omega_{Q} / \omega_{z}\right)^{2}=3$ the BEC is in the regime of $1 \mathrm{D}$ confinement and mean-field bosons (TF 1D), and at $\left(\omega_{Q} / \omega_{z}\right)^{2}=4$ the BEC is in the regime of $1 \mathrm{D}$ confinement and quasi-ideal bosons (free bosons 1D). Thus Figure 2 shows that it is necessary a very strong anisotropy $\lambda$ to reach the regime of $1 \mathrm{D}$ confinement and mean-field bosons, and $\omega_{Q}$ is able to discriminate between this regime and that of $1 \mathrm{D}$ confinement and quasi-ideal bosons. Note that, as shown in [10], the Tonks regime is avoided if $N\left(a_{s} / a_{H}\right) \gg \lambda^{5 / 3}\left(a_{s} / a_{H}\right)^{3}$ : the results of Fig. 2 are reliable for all values of horizontal axis under the condition $a_{s} / a_{H} \ll \lambda^{-5 / 9} 10^{-10 / 3}$.

Our variational approach can be applied also to the 
case of an attractive BEC $\left(a_{s}<0\right)$. In this case it exists a critical inter-atomic strength below which there is the collapse of the BEC [11], as shown in Figure 3.
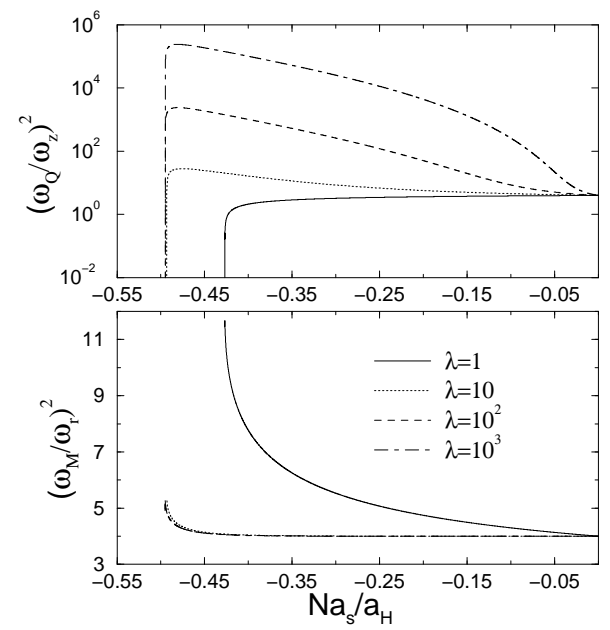

FIG. 3: Monopole frequency $\omega_{M}$ and quadrupole frequency $\omega_{Q}$ of collective oscillations for a cigar-shaped BEC. Case of negative scattering length $a_{s}$.

In the spherical case the monopole frequency $\omega_{M}$ grows by decreasing the interaction strength up to the collapse, but it is sufficient a small anisotropy to freeze $\omega_{M}$ to a constant value $\left(\omega_{M} / \omega_{r}\right)^{2}=4$. Instead the quadrupole frequency $\omega_{Q}$ strongly increases with $\lambda$ but always goes to zero at the collapse.

From 3D to 2D. - The dynamics of a disc-shaped BEC of $N$ atoms, confined by a harmonic potential of frequency $\omega_{z}$ in the cylindric axial direction $z$ and by a generic potential $W(x, y)$ in the cylindric radial direction $(x, y)$, is well described by an effective $2 \mathrm{D}$ nonpolynomial Schrödinger equation (2D NPSE) for the radial wave function $\phi(x, y, t)$ of the BEC. Within the $2 \mathrm{D}$ NPSE approach, the BEC is approximated by a Gaussian of width $\eta$ in the cylindric axial direction, given by $\eta^{4}-\gamma N|\phi|^{2} \eta-1=0$, where $\gamma=2 \sqrt{2 \pi} a_{s} / a_{z}$ is the effective interaction strength and $a_{z}=\sqrt{\hbar /\left(m \omega_{z}\right)}$ the characteristic harmonic length in the radial direction. Here length is in units $a_{z}$ and energy in units $\hbar \omega_{z}$.

Note that in the limit of weak axial confinement, which corresponds to the $3 \mathrm{D}$ regime, the radial density $N|\phi|^{2}$ satisfies the condition $\gamma N|\phi|^{2}>>1$ and the 2D NPSE reduces to a $2 \mathrm{D}$ nonlinear Schrödinger equation with quadratic nonlinearity. In the opposite limit $\gamma N|\phi|^{2}<<1$ of strong axial confinement, which corresponds to the $2 \mathrm{D}$ regime, the $2 \mathrm{D}$ NPSE becomes a $2 \mathrm{D}$ nonlinear Schrödinger equation with cubic nonlinearity, also called 2D GPE [7].

In the case $W(x, y)=0$, which corresponds to a homogeneous BEC in the cylindrical radial direction with periodic boundary conditions, the chemical potential reads

$$
\mu=\gamma \frac{\rho_{2}}{\eta}+\frac{1}{4}\left(\frac{1}{\eta^{2}}+\eta^{2}\right)=\frac{5}{4} \eta^{2}-\frac{3}{4 \eta^{2}} .
$$

It is easy to show that Eq. (4) reduces to $\mu=$ $(5 / 4)(g \rho)^{1 / 2}$ in the $3 \mathrm{D}$ regime $\left(\eta=\left(\gamma \rho_{2}\right)^{1 / 3}\right)$ and it becomes $\mu=g \rho_{2}+1 / 2$ in the $1 \mathrm{D}$ regime $(\eta=1)$. The term $1 / 2$ is the axial energy in scaled units. From the 2D NPSE one calculates the radial velocity $c_{2}$ of first sound as $c_{2}=\left(\rho_{2} \frac{\partial \mu}{\partial \rho_{2}}\right)^{1 / 2}$.

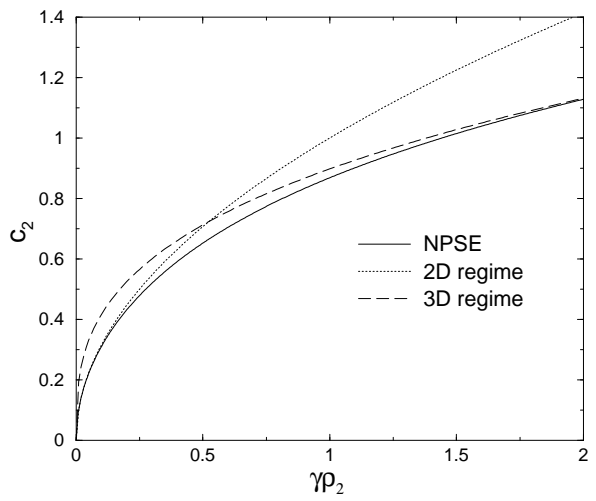

FIG. 4: First sound radial velocity $c_{2}$ versus inter-atomic strength $\gamma \rho_{2}$, where $\gamma=2 \sqrt{2 \pi} a_{s} / a_{z}$ with $a_{s}$ scattering length and $a_{z}$ radial harmonic length. $\rho_{2}$ is the radial density of the disc-shaped radially-homogeneous BEC. Length in units $a_{z}$, density in units $1 / a_{z}^{2}$ and time in units $1 / \omega_{z}$.

In Figure 4 we plot $c_{2}$ as a function of $\gamma \rho_{2}$. The velocity of sound is $c_{2}=(5 / 6)\left(\gamma \rho_{2}\right)^{1 / 3}$ in the $3 \mathrm{D}$ regime and it becomes $c_{2}=\sqrt{\gamma \rho_{2}}$ in the $2 \mathrm{D}$ regime. Our $2 \mathrm{D}$ value for $c_{2}$ is in full agreement with the result obtained from 2D GPE in the TF approximation, while the 3D value of $c_{2}$ differs from the exact one, obtained from the 3D GPE, only for the constant that is $3^{2 / 3} \pi^{1 / 3} / 2^{7 / 3}$ instead of $5 / 6$. This error (less than $2 \%$ ) in the $3 \mathrm{D}$ regime is a consequence of using in the 2D NPSE a Gaussian axial wave function. Thus, our sound velocity $c_{2}$ interpolates remarkably well between the exact results of low density (2D regime) and high density (3D regime).

In the case $W(x, y)=m \omega_{r}^{2}\left(x^{2}+y^{2}\right) / 2$ the collective oscillations of the BEC can be obtained from Eq. (3) with the condition of disc-shaped BEC, i.e. $0<\lambda<1$. In Figure 5 we plot monopole and quadrupole frequencies of the BEC with a weak anisotropy. The monopole frequency $\omega_{M}$ does not depend on $\lambda$ in the limit of very small inter-atomic strength and coincides with the ideal gas limiting value. In the regime of mean-field bosons, $\omega_{M}$ changes with $\lambda$ from $\left(\omega_{M} / \omega_{z}\right)^{2}=5$ (isotropic TF 3D) to $\left(\omega_{M} / \omega_{z}\right)^{2}=3$ (anisotropic TF 3D). In a similar way the quadrupole frequency $\omega_{Q}$ moves from $\left(\omega_{Q} / \omega_{r}\right)^{2}=2$ (isotropic TF 3D) to $\left(\omega_{Q} / \omega_{r}\right)^{2}=10 / 3$ (anisotropic TF $3 \mathrm{D})$, as predicted by Stringari [12]. 


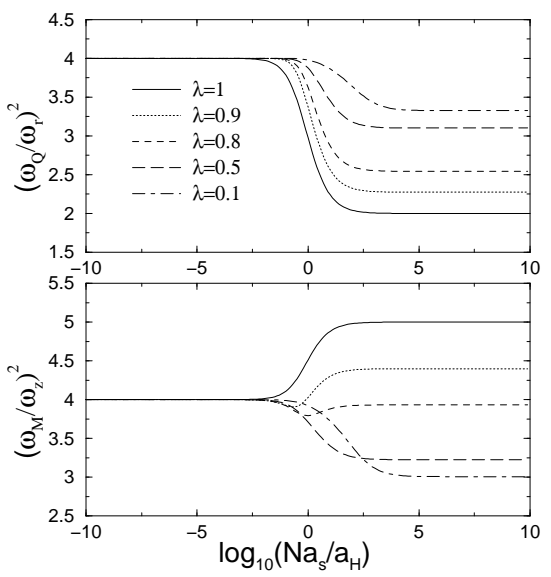

FIG. 5: Monopole frequency $\omega_{M}$ and quadrupole frequency $\omega_{Q}$ of collective oscillations for a disc-shaped BEC. Case of positive scattering length $a_{s}$ with small anisotropy.

By further increasing the disc-shaped anisotropy the main effect is that of shifting the transition from the ideal gas limit to the 3D regime at higher values of the interaction strength. As shown in Figure 6, both monopole and quadrupole frequencies display this behavior. Thus, these frequencies do not discriminate the regime of $2 \mathrm{D}$ confinement and mean-field bosons from the regime of 2D confinement and quasi-ideal bosons.

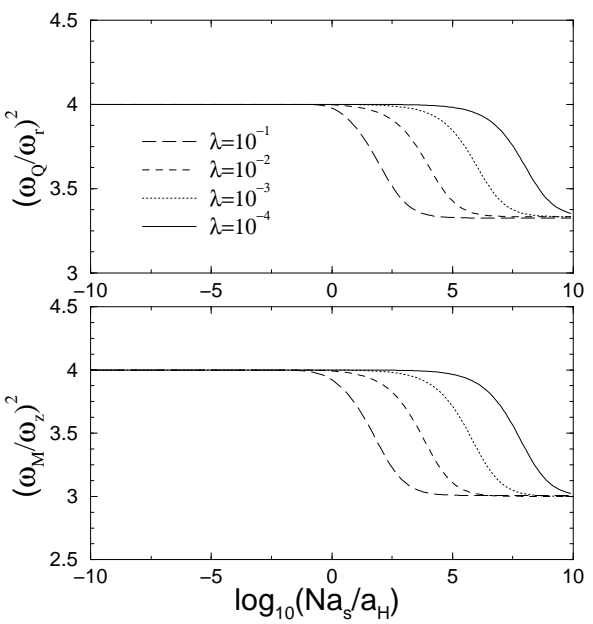

FIG. 6: Monopole frequency $\omega_{M}$ and quadrupole frequency $\omega_{Q}$ of collective oscillations for a disc-shaped BEC. Case of positive scattering length $a_{s}$ with large anisotropy.

The frequencies simply give a signature of the transition from ideal gas to TF 3D. Clearly, under strong confinement it is necessary to increase the inter-atomic interaction to mantain the $3 \mathrm{D}$ regime. Note that for the $\mathrm{TF}$ $2 \mathrm{D}$ regime the dispersion relation of collective frequencies can be analytically obtained using the same hydrodynamical approach applied by Stringari for the TF 1D regime [12]. In this way one recovers the result $\omega^{2}=4 \omega_{r}^{2}$ for the quadrupole frequency.

The calculations with negative scattering length $a_{s}$ suggest that monopole and quadrupole oscillations of a highly deformed disc-shaped BEC do not depend on the inter-atomic strength.

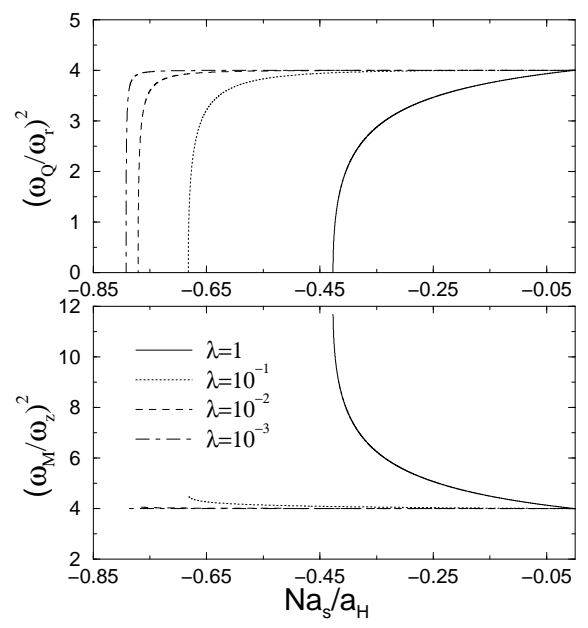

FIG. 7: Monopole frequency $\omega_{M}$ and quadrupole frequency $\omega_{Q}$ of collective oscillations for a disc-shaped BEC. Case of negative scattering length $a_{s}$.

As shown in Figure 7, only very close to the collapse $\omega_{Q}$ goes to zero while $\omega_{M}$ remains essentially constant.

In conclusion, our results exactly reproduces the ones obtained in specific dimensional regimes with the hydrodynamic method or the sum-rule method, but, they also give the crossover from one regime to another.

[1] A. Gorlitz et al., Phys. Rev. Lett. 87, 080403 (2001).

[2] F. Schreck et al., 87, 080403 (2001).

[3] M. Olshanii, Phys. Rev. Lett. 81, 938 (1998).

[4] A.D. Jackson, G.M. Kavoulakis and C.J. Pethick, Phys. Rev. A 58, 2417 (1998).

[5] M.L. Chiofalo and M.P. Tosi, Phys. Lett. A 268, 406 (2000).

[6] D.S. Petrov, G.V. Shlyapnikov, and J.T.M. Walraven, Phys. Rev. Lett. 84, 2551 (2000).

[7] L. Salasnich, A. Parola, and L. Reatto, Phys. Rev. A 65, 043614 (2002); L. Salasnich, Laser Phys. 12, 198 (2002); 13, 543 (2003).

[8] V.M. Perez-Garcia et al., Phys. Rev. Lett. 77, 5320 (1996); L. Salasnich, Int. J. Mod. Phys. B 14, 1 (2000).

[9] L. Tonks, Phys. Rev. 50, 955 (1936); M. Girardeau, J. Math. Phys. 1, 516 (1960); E.H. Lieb and W. Liniger, Phys. Rev. 130, 1605 (1963).

[10] C. Menotti and S. Stringari, Phys. Rev. A 66, 043610 (2002).

[11] P.A. Ruprecht et al. Phys. Rev. A 51, 4704 (1995).

[12] S. Stringari, Phys. Rev. A 58, 2385 (1998). 